Editorial

\title{
Molecular Imaging and Radiotherapy: Theranostics for Personalized Pa- tient Management
}

\author{
Irina Velikyan ${ }^{\bowtie}$ \\ PET-Centre, Centre for Medical Imaging, Uppsala University Hospital, SE-751 85 Uppsala, Sweden
}

$\bowtie$ Corresponding author: Tel: +46 (0) 18611 0654; Cell phone: +46 704834137; E-mail: irina.velikyan@bms.uu.se

(C) Ivyspring International Publisher. This is an open-access article distributed under the terms of the Creative Commons License (http://creativecommons.org/ licenses/by-nc-nd/3.0/). Reproduction is permitted for personal, noncommercial use, provided that the article is in whole, unmodified, and properly cited.

Received: 2012.04.01; Accepted: 2012.04.01; Published: 2012.05.01

\begin{abstract}
This theme issue presents current achievements in the development of radioactive agents, pre-clinical and clinical molecular imaging, and radiotherapy in the context of theranostics in the field of oncology.
\end{abstract}

Key words: PET, SPECT, Radiotherapy, nuclear medicine, receptor, theranostics

Nuclear medicine is an expanding field for the diagnostics and therapy on a molecular level. Current nuclear medicine diagnostic techniques include non-invasive Positron Emission Tomography (PET) and Single Photon Emission Computed Tomography (SPECT) utilizing, respectively, positron and gamma emitting radionuclides for the generation of the signal. Both external and internal radiotherapy can be directed by diagnostic PET and SPECT, and diagnostic imaging and radiotherapy are merging into theranostics resulting in more personalized medicine. For example, the individualized diagnosis is becoming a standard in the selection of patients for peptide receptor radionuclide therapy wherein the pre-therapeutic imaging and the radiotherapy are conducted with the same vector molecule by exchanging the imaging and therapeutic radionuclides. The high value of imaging diagnostics in assisting cancer therapy in terms of early detection, staging, therapy selection and planning as well as follow-up is recognized.

Development of molecular imaging and radiotherapeutical agents is a complex process including identification of the biological target, respective lead compound, synthesis of the radioactive agent, its chemical characterization, pre-clinical, and clinical evaluation. Nevertheless, the number of new agents for targeted imaging of specific protein expression products (receptors, enzymes, and antigens), pre-targeted imaging using small effector or hapten molecules as well as non-targeted imaging of pulmonary and myocardial perfusion and ventilation is increasing continuously. Imaging of inflammation, infection as well as general downstream biologic properties such as proliferation, hypoxia, glycolysis, and angiogenesis has also been investigated. The introduction of new radiopharmaceuticals and their accessibility are important factors determining the expansion of clinical nuclear medicine for early disease detection and personalized medicine with higher therapeutic efficiency. This issue is focused on the targeted and pre-targeted imaging and radiotherapy in oncology.

The role of molecular imaging in the individualized patient management is hard to overestimate and it is reflected in the increasing number of specifically acting imaging agents entering clinical nuclear medicine as well as expansion of diverse applications 
of established [18F]flurodeoxyglucose. The review by Sörensen [1] is dedicated to the exploration of the contribution of clinical PET to the improved and personalized treatment of patients. Diversity of clinical PET imaging agents as well as technical and regulatory aspects are thoroughly discussed. The review provides valuable and comprehensive description of considerable number of clinically relevant imaging agents.

Molecular imaging and therapy has been integrated and most thoroughly studied for the management of patients affected by neuroendocrine tumors (NETs) targeting somatostatin receptors (SSTRs). Therefore, several papers in this issue cover various aspects of this topic. Two Centers of excellence recognized by European Neuroendocrine Tumor Society, Zentralklink Bad Berka, Germany and Uppsala University Hospital, Sweden share with us their valuable experience in personalized medicine closely combining diagnostic imaging and therapy. Baum et al. [2] provide a comprehensive introduction of Theranostics concept and report on pioneer and diverse applications of various imaging and therapeutic agents in oncology. Öberg [3] reviews various imaging and radiotherapeutic agents as well as targets that can be utilized for the diagnosis and treatment of NETs in the context of personalized medicine. Specific example of an outstanding case patient study is presented by Garske et al. [4]. The importance of ${ }^{177} \mathrm{Lu}$ radionuclide (in the form of ${ }^{177} \mathrm{Lu}$-DOTA-octreotate) for the treatment of patients with highly proliferating large NETs is considered and highly appreciated. This report also discusses the crucial role of imaging and dosimetry in the selection, planning, and response monitoring of the treatment. The clinical study including 112 patients conducted by Delpassand et al. [5] gives an updated perspective on the value of ${ }^{111} \mathrm{In}$ radionuclide for the therapeutical applications. The results demonstrate that the high dose ${ }^{111}$ In-Pentetreotide is a safe and effective therapy means for patients with disseminated NETs.

The pioneer studies of SSTRs triggered an accelerated development of other small peptide based agents targeted to the respective receptors. Fani et al. [6] review the current status of somatostatin, bombesin, vasoactive intestinal peptide, gastrin, neurotensin, exendin and RGD based analogues developed for PET and SPECT imaging as well as for the radiotherapy applications in oncology. Imaging agents for monitoring general downstream biologic property such as angiogenesis are reviewed by Backer et al. [7] with focus on integrins and receptors for vascular endothelial growth factor (VEGF). The key application of the respective radiolabeled imaging ligands is monitoring response to anti-angiogenic therapy and providing image-guided therapy.

Another class of specific protein expression products is enzymes that can be visualized by radiolabeled enzyme inhibitors. Sundin [8] reviews the imaging agent arsenal used in the management of adrenocortical cancer with the focus on ${ }^{11} \mathrm{C}$-metomidate that binds with high specificity and affinity to $11-\beta$-hydroxylase providing the only means for the differentiation of benign and malignant adrenocortical tumors from those of non-adrenocortical origin. This particular example stresses the importance of the development of agents specific to tumor characteristics.

The current status of the pre-targeted imaging and therapy using combination of a bispecific antibody and radiolabeled hapten molecule is thoroughly reviewed by Goldenberg et al. [9]. The pre-targeting concept provides a means to overcome the drawbacks related to slow pharmacokinetics and prolonged blood circulation of labeled antibodies resulting in high radiation dose to the healthy tissue and poor contrast image. A number of bispecific antibodies targeting various tumor types have been developed and some entered clinical trials.

In summary, this theme issue presents current achievements in the development of radioactive agents, pre-clinical and clinical molecular imaging and radiotherapy in the context of theranostics in the field of oncology. The significance of the targeted and pre-targeted imaging using PET and SPECT for the individualized treatment management and monitoring therapy response is discussed by the authors. The content of the issue reflects the worldwide appreciation of theranostics and its accelerated implementation in nuclear medicine with the ultimate aim for personalized medicine.

The authors are immensely appreciated for sharing their valuable experience, knowledge, and perception and contributing to the expansion of clinical nuclear medicine and theranostics.

\section{Competing Interests}

The author has declared that no conflict of interest exists.

\section{References}

1. Sörensen J. How does the patient benefit from clinical PET? Theranostics 2012; 2: 427-36.

2. Baum RP, Kulkarni HR. THERANOSTICS: From Molecular Imaging Using Ga-68 Labelled Tracers and PET/CT to Personalized Radionuclide Therapy - The Bad Berka Experience. Theranostics 2012; 2: $437-47$.

3. Öberg K. Molecular Imaging and Radiotherapy: Theranostics for Personalized Patient Management of Neuroendocrine Tumors (NETs). Theranostics 2012; 2: 448-58. 
4. Garske U, Sandström M, Johansson S, Granberg D, Lundqvist H, Lubberink M, Sundin A, Eriksson B. Lessons on tumour response: Imaging during therapy with ${ }^{177} \mathrm{Lu}$-DOTA-octreotate. A case report on a patient with a large volume of a poorly differentiated neuroendocrine tumour. Theranostics 2012; 2: 459-71.

5. Delpassand ES, Samarghandi A, Mourtada JS, Zamanian S, Espanan G, Sharif R, MacKenzie S, Kosari K, Barakat O, Naqvi Sh, Seng JE, Anthony L. Long-Term Survival, Toxicity Profile, and role of F-18 FDG PET/CT scan in Patients with Progressive Neuroendocrine Tumors Following Peptide Receptor Radionuclide Therapy with High Activity In-111 Pentetreotide. Theranostics 2012; 2: 472-80.

6. Fani M, Maecke HR, Okarvi SM. Radiolabeled Peptides: Potential tools for the detection and treatment of cancer. Theranostics 2012; 2: 481-501.

7. Backer MV, Backer JM. Imaging Key Biomarkers of Tumor Angiogenesis. Theranostics 2012; 2: 502-15.

8. Sundin A. Imaging of Adrenal Masses with Emphasis on Adrenocortical Tumors. Theranostics 2012; 2: 516-22.

9. Goldenberg DM, Chang ChH, Rossi EA, McBribe WJ, Sharkey RM. Pre-targeted molecular imaging and radioimmunotherapy. Theranostics 2012; 2: 523-40. 